\title{
Research on Evolution Dynamics of Urban Rail Transit Network Based on Allometric Growth Relationship
}

\author{
Zehua Zhang ${ }^{1}$, Shumin Feng ${ }^{1}$, Huihui $\mathrm{Jia}^{2}, \mathrm{Hao} \mathrm{Liu}^{1}$, Chao Yang ${ }^{3}$, and Maohua Kang ${ }^{4}$ \\ ${ }^{1}$ Harbin Institute of Technology \\ ${ }^{2}$ SEGi University \\ ${ }^{3}$ Heilongjiang Institute of Technology \\ ${ }^{4}$ Guang Dong Peizheng College
}

February 7, 2022

\begin{abstract}
There are different dynamic characteristics in each stage of rail transit network development. It can provide guidance for rail transit network planning and phased construction,Evaluating the evolution degree of urban rail transit network and accurately dividing the evolution stages. Starting from the allometric growth relationship between transfer nodes and ordinary nodes in urban rail transit network, this paper defines the evolution level of rail transit network and the growth rate difference between transfer and ordinary nodes, and deduces the logistic dynamic equation of urban rail transit network evolution level with the help of mathematical derivative and logarithmic relationship, This paper reveals the dynamic law of the evolution process of urban rail transit network from the theoretical level, analyzes the evolution dynamic process on this basis, finds the Threshold for phasing the evolution stage, and divides it into four evolution stages. Finally, the theoretical derivation results are verified with the evolution data of Beijing Rail Transit Network (1984-2020) for 45 years. The verification results are highly consistent with the theoretical derivation results, which proves the correctness of the theoretical model and its practical guiding significance for the construction of rail transit network.
\end{abstract}

\section{Hosted file}

Evolution Dynamics Based on Allometric Growth2021216.docx available at https://authorea. com/users/459236/articles/555560-research-on-evolution-dynamics-of-urban-rail-transitnetwork-based-on-allometric-growth-relationship 